\title{
IKERLANAK
}

\section{MONOTONIC CORE SOLUTIONS: BEYOND YOUNG'S THEOREM}

by

Javier Arin

2010

Working Paper Series: IL. 44/10

Departamento de Fundamentos del Análisis Económico I

Ekonomi Analisiaren Oinarriak I Saila

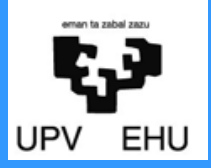

University of the Basque Country 


\title{
Monotonic core solutions: Beyond Young's theorem
}

\author{
J. Arin*
}

July 26, 2010

\begin{abstract}
We introduce two new monotonicity properties for core concepts: singlevalued solution concepts that always select a core allocation whenever the game is balanced (has a nonempty core). We present one result of impossibility for one of the properties and we pose several open questions for the second property. The open questions arise because the most important core concepts (the nucleolus and the per capita nucleolus) do not satisfy the property even in the class of convex games.
\end{abstract}

Keywords: Monotonicity, Core, TU games, nucleolus per capita

*Dpto. Ftos. A. Económico I, Basque Country University, L. Agirre 83, 48015 Bilbao, Spain. Email: franciscojavier.arin@bs.ehu.es. J. Arin acknowledges financial support from Project 9/UPV00031.321-15352/2003 of the University of the Basque Country, Projects BEC2003-08182 and SEJ-2006-05455 of the Ministry of Education and Science of Spain and Project GIC07/146-IT-377-07 of the Basque Goverment. Likewise, 


\section{Introduction}

Young (1985) formulates an impossibility result for the problem of finding core concepts satisfying monotonicity ${ }^{1}$ in the domain of balanced TU games. This result opens up two paths of research: One is to restrict the search for monotonic core concepts to certain classes of games ${ }^{2}$. The other, is to define new properties of monotonicity (weaker than the one formulated by Young) and to deal with the class of all TU balanced games. This paper discusses this second path.

In considering new monotonicity properties we first analyze the compatibility between monotonicity and core belonging. Only when such a compatibility exists do we require a core concept to be monotonic. Following this approach we introduce two new properties: strong core monotonicity and core monotonicity. For the first property we provide a result of impossibility. For the second property we pose an open question once we have checked that core concepts such as nucleolus and per capita nucleolus do not satisfy the property.

\section{Preliminaries}

\section{$2.1 \quad$ TU Games}

A cooperative $n$-person game in characteristic function form is a pair $(N, v)$, where $N$ is a finite set of $n$ elements and $v: 2^{N} \rightarrow \mathbb{R}$ is a real-valued function in the family $2^{N}$ of all subsets of $N$ with $v(\emptyset)=0$. Elements of $N$ are called players and the real-valued function $v$ the characteristic function of the game. Any subset $S$ of $N$ is called a coalition. The number of players in $S$ is denoted by $|S|$. Given $S \subset N$ we denote by $N \backslash S$ the set of players of $N$ that are not in $S$. Let $N$ be a set of players and $\Gamma_{0}$ a class of games. If there is no confusion, we write $v \in \Gamma_{0}$ instead of $(N, v) \in \Gamma_{0}$. A distribution of $v(N)$ among the players is a real-valued vector $x \in \mathbb{R}^{N}$ where $x_{i}$ is the payoff assigned by $x$ to player $i$. A distribution satisfying $x_{i} \geq v(i)$ for all $i \in N$ is called an imputation and the set of imputations is denoted by $I(v)$. We denote $\sum_{i \in S} x_{i}$ by $x(S)$. The core of a game is the set of imputations that cannot be blocked by any coalition, i.e.

$$
C(v)=\{x \in I(v): x(S) \geq v(S) \text { for all } S \subset N\} .
$$

\footnotetext{
${ }^{1}$ See Section 2 and 3 for formal definitions of core concept and monotonicity.

${ }^{2}$ This also can be seen as a weakening of the core requirements in the sense that we only search in games that have a special core.
} 
A game with a non-empty core is called a balanced game. Player $i$ is a veto player if $v(S)=0$ for all $S$ where player $i$ is not present. A balanced game with at least one veto player is called a veto balanced game. We denote by $\Gamma_{B}$ the class of balanced games and by $\Gamma_{B V}$ the class of veto balanced games.

A solution $\phi$ in a class of games $\Gamma_{0}$ is a correspondence that associates a set $\phi(N, v)$ in $\mathbb{R}^{N}$ with every game $(N, v)$ in $\Gamma_{0}$ such that $x(N) \leq v(N)$ for all $x \in \phi(N, v)$. This solution is efficient if this inequality holds with equality. The solution is single-valued if the set contains a unique element for each game in the class.

Given $x \in \mathbb{R}^{N}$ the excess of a coalition $S$ with respect to $x$ in a game $v$ is defined as $e(S, x):=v(S)-x(S)$. Let $\theta(x)$ be the vector of all excesses at $x$ arranged in non-increasing order. The lexicographic order $\prec_{L}$ between two vectors $x$ and $y$ is defined by $x \prec_{L} y$ if there exists an index $k$ such that $x_{l}=y_{l}$ for all $l<k$ and $x_{k}<y_{k}$ and the weak lexicographic order $\preceq_{L}$ by $x \preceq_{L} y$ if $x \prec_{L} y$ or $x=y$.

Schmeidler (1969) introduced the nucleolus of a game $v$, denoted by $\eta(v)$, as the unique imputation that lexicographically minimizes the vector of non increasingly ordered excesses over the set of imputations. In formula:

$$
\{\eta(N, v)\}=\left\{x \in I(N, v) \mid \theta(x) \preceq_{L} \theta(y) \text { for all } y \in I(N, v)\right\} \text {. }
$$

For any game $v$ with a non-empty imputation set, the nucleolus is a singlevalued solution, is contained in the kernel and lies in the core provided that the core is non-empty.

The per capita nucleolus is defined analogously by using the concept of per capita excess instead of excess. Given $S$ and $x$ the per capita excess of $S$ at $x$ is

$$
e^{p c}(S, x):=\frac{v(S)-x(S)}{|S|}
$$

In this paper, we study solution concepts that select precisely one core allocation for each balanced game. We call such concepts core concepts.

\subsection{On monotonicity properties}

We present some monotonicity properties for single-valued solution concepts. 
Let $v, w \in \Gamma_{0}$, such that for all $T$ containing player $i, v(T) \leq w(T)$, and for all $S \subseteq N \backslash\{i\} v(S)=w(S)$ : We say that game $w$ is monotonic with respect to game $v$ and player $i$.

Let $\phi$ be a single-valued solution in a class of games $\Gamma_{0}$.

We say that solution $\phi$ satisfies monotonicity if for all $v, w \in \Gamma_{0}$, such that $w$ is monotonic with respect to game $v$ and player $i$ then $\phi_{i}(w) \geq \phi_{i}(v)$.

We say that solution $\phi$ satisfies N-monotonicity (Meggido, 1974) if for all $v, w \in \Gamma_{0}$, such that for all $S \neq N, v(S)=w(S)$ and $v(N)<w(N)$, then for all $i \in N, \phi_{i}(v) \leq \phi_{i}(w)$.

We say that solution $\phi$ satisfies strong $N$-monotonicity if for all $v, w \in \Gamma_{0}$, such that for all $S \neq N, v(S)=w(S)$ and $v(N)<w(N)$, then for all $i, j \in N$, $\phi_{i}(w)-\phi_{i}(v)=\phi_{j}(w)-\phi_{j}(v) \geq 0$.

Clearly, monotonicity implies N-monotonicity.

Young (1985) shows that in the class of balanced games there is no core concept that satisfies monotonicity. The result is presented by means of two balanced games with only one core allocation each. Therefore in these games a core concept must choose the allocation contained in the core. To some extent, we can say that in this case the core restrictions do not allow a core concept to be monotonic. This observation motivates the following note. We investigate new monotonicity properties that require for some compatibility between monotonicity and core belonging.

In the class of convex games the Shapley value is a core concept that satisfies monotonicity. Arin and Feltkamp (2007) show that in the class of veto balanced games there are several core concepts that satisfy monotonicity. It is also shown that the nucleolus and the per capita nucleolus do not satisfy the property.

\section{On core monotonicity}

We introduce new core monotonicity properties ${ }^{3}$. The idea of the new properties is that a core concept should be monotonic whenever core restrictions are compatible with this monotonicity.

Definition 1 Let $v, w \in \Gamma_{0}$, such that game $w$ is monotonic with respect to game $v$ and player $i$. We say that game $w$ has a monotonic core with respect to

\footnotetext{
${ }^{3}$ We define the properties for core concepts.
} 
game $v$ and player $i$ if there exist $x \in C(w)$ and $z \in C(v)$ such that $x_{i} \geq z_{i}$.

The existence of a monotonic core is certainly a necessary condition for the monotonicity of a core concept. If this requirement is not satisfied any core concept must violate monotonicity. In the example given by Young the requirement is not satisfied.

Following this observation we define strong core monotonicity.

Definition 2 Strong core monotonicity: A core concept $\phi$ in $\Gamma_{0}$ is strongly core monotonic if for all $v, w \in \Gamma_{0}$ such that:

1.- game $w$ is monotonic with respect to game $v$ and player $i$

2.- game $w$ has a monotonic core with respect to game $v$ and player $i$

it holds that, $\phi_{i}(w) \geq \phi_{i}(v)$.

Note that if a core concept is monotonic then is strongly core monotonic.

Theorem 3 In the class of balanced games there is no core concept that satisfies strong core monotonicity.

Proof. Let $N=\{1,2,3,4\}$ a set of players and consider the following 4person balanced games:

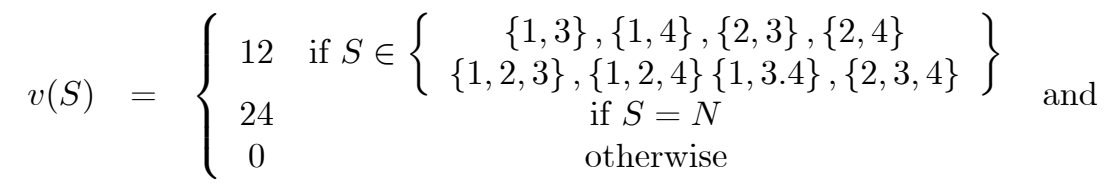

$$
\begin{aligned}
& w^{i}(S)=\left\{\begin{array}{cl}
v(S) & \text { if } S \neq N \backslash\{i\} \\
24 & \text { if } S=N \backslash\{i\}
\end{array}\right.
\end{aligned}
$$

where $i \in N$. The games $w^{i}$ are monotonic with respect to to game $v$ and player $l, l \neq i$. They also have a monotonic core with respect to game $v$ and player $l$, $l \neq i$. For the games $w^{1}$ and $w^{2}$ we need to consider the only core allocation of the two games, $z^{1}=(0,0,12,12)$ and for games $w^{3}$ and $w^{4}$ we need to consider the allocation $z^{3}=(12,12,0,0)$.

Let $\phi$ a core concept satisfying strong core monotonicity. By applying strong core monotonicity between games $w^{1}$ and $v$ we conclude that $\phi(v)=z^{1}$. By applying strong core monotonicity between games $w^{3}$ and $v$ we conclude that $\phi(v)=z^{3}$.

In this example, core restrictions do not allow a core concept to behave monotonically from game $v$ to games $w^{1}$ and $w^{3}$. We try to find a definition of 
core monotonicity that requires monotonic behavior of a core concept whenever core restrictions allow such behavior.

Let $N$ be a set of players and $\Gamma_{0}$ a class of games. By a pair $(w, i(w))$ we refer to a game $w \in \Gamma_{0}$ and a player $i \in N$ associated to game $w$.

Definition 4 Let $N$ be a set of players and $v \in \Gamma_{0}$. We say that game $v$ has an extendable monotonic core if there exists $z \in C(v)$ such that for any pair $(w, i(w))$ where $w$ is a monotonic game with respect to $v$ and $i(w)$ we can find $x \in C(w)$ such that $x_{i(w)} \geq z_{i(w)}$.

In the class of convex games any game has an extendable monotonic core since, in this class, the Shapley value is a monotonic core concept.

In the above proof it is immediate that game $v$ does not have an extendable monotonic core in the class of all balanced games. The following property incorporates this new aspect of the compatibility between monotonicity and core belonging.

Definition 5 Extendable core monotonicity: A core concept $\phi$ on $\Gamma_{0}$ is extendable core monotonic if for all $v, w \in \Gamma_{0}$ such that:

1.- game $w$ is monotonic with respect to game $v$ and player $i$

2.- game $w$ has a monotonic core with respect to game $v$ and player $i$

3. $-\phi_{i}(w)<\phi_{i}(v)$

then game $v$ does not have an extendable monotonic core.

This definition is not completely satisfactory as the following example illustrates $^{4}$.

Let $N=\{1,2,3.4\}$ a set of players and consider the following 4-person balanced games:

$$
\begin{aligned}
& v(S)=\left\{\begin{array}{cc}
12 & \text { if } S \in\left\{\begin{array}{c}
\{1,3\},\{1,4\},\{2,3\},\{2,4\} \\
\{1,2,3\},\{1,2,4\}\{1,3.4\},\{2,3,4\}
\end{array}\right\} \\
24 & \text { of } S=N \\
0 & \text { otherwise }
\end{array}\right. \text { and } \\
& q(S)=\left\{\begin{array}{cc}
v(S) & \text { if } S \neq N \\
28 & \text { if } S=N
\end{array} .\right.
\end{aligned}
$$

It seems clear that a core concept $\phi$ such that $\phi_{i}(v)>\phi_{i}(q)$ for a player $i$ cannot be considered as a monotonic core concept. But since game $v$ has no

\footnotetext{
${ }^{4}$ The property does not imply $N$-monotonicity. That is, a solution can be monotonic in the sense of Definition 5 but violates $N$-monotonicity.
} 
extendable monotonic core according to the definition above, we cannot claim that this solution $\phi$ is not extendable core monotonic using the above facts.

In this example, it does not seem reasonable to argue that game $v$ has no extendable monotonic core in order to justify non-monotonic behavior of a core concept while moving from game $v$ to game $q$. For such an argument, it seems necessary to show that game $q$ is necessary to imply that game $v$ has no extendable monotonic core. This idea is formalized in the property we call core monotonicity.

Definition 6 Let $v \in \Gamma_{0}$. We say that game $v$ has an extendable monotonic core with respect to the list of pairs $\left(w^{i}, j\left(w^{i}\right)\right)_{i=1, \ldots, k}$ if:

1.- for any pair $\left(w^{i}, j\left(w^{i}\right)\right)$ it holds that $w^{i} \in \Gamma_{0}$ and $w^{i}$ is a monotonic game with respect to $v$ and $j\left(w^{i}\right)$.

2.- there exists $z \in C(v)$ such that for any pair $\left(w^{i}, j\left(w^{i}\right)\right)$ we can find $x \in C\left(w^{i}\right)$ such that $x_{j\left(w^{i}\right)} \geq z_{j\left(w^{i}\right)}$.

Note that, in the proof of Theorem 3, game $v$ has no extendable monotonic core with respect to the list of pairs: $\left(w^{1}, 2\right),\left(w^{2}, 1\right),\left(w^{4}, 3\right)$ and $\left(w^{3}, 4\right)$.

Definition 7 Core monotonicity: A core concept $\phi$ in $\Gamma_{0}$ is core monotonic if for all $v, w \in \Gamma_{0}$ such that:

1.- game $w$ is monotonic with respect to game $v$ and player $i$

2.- game $w$ has a monotonic core with respect to game $v$ and player $i$

3.- $\phi_{i}(w)<\phi_{i}(v)$

then there exists a list of pairs $\left(w^{l}, j\left(w^{l}\right)\right)_{l=1, \ldots, k}$, such that

1.- game $v$ has an extendable monotonic core for the list of pairs $\left(w^{l}, j\left(w^{l}\right)\right)_{l=1, \ldots, k}$.

2,.- game $v$ has no extendable monotonic core for the list of pairs $(w, i)$, $\left(w^{l}, j\left(w^{l}\right)\right)_{l=1, \ldots, k}$.

The property means that any violation of the requirement of monotonicity should be justified. If such a violation occurs between two games, from game $v$ to game $w$, then game $w$ should be a member of a minimal list of games for which game $v$ has no extendable monotonic core. Minimality implies that for any other subset of the list of games, game $v$ has an extendable monotonic core.

Remark 8 If a core concept is strongly core monotonic then is core monotonic. 
In the class of balanced games there exist core concepts that satisfy Nmonotonicity such as the per capita nucleolus. Therefore core restrictions and Nmonotonicity are compatible. The following proposition relates core monotonicity and N-monotonicity.

Proposition 9 Core monotonicity implies N-monotonicity.

Proof. Assume that there exists a core concept $\phi$ that is core monotonic but not N-monotonic. Then there exist two balanced games $(N, v)$ and $(N, w)$ such that:

1.- $w(N)>v(N)$ and $w(S)=v(S)$ for any other coalition $S$.

2.- game $w$ has a monotonic core with respect to game $v$ and player $i$

3.- $\phi_{i}(w)<\phi_{i}(v)$

4.- there exists a list of pairs $\left(\left(N, q^{l}\right), j\left(q^{l}\right)\right)_{l=1, \ldots, k}$, such that:

a.- game $v$ has an extendable monotonic core for the list of pairs $\left(\left(N, q^{l}\right), j\left(q^{l}\right)\right)_{l=1, \ldots, k}$.

b.- game $v$ has not an extendable monotonic core for the list of pairs $((N, w) . i)$, $\left(\left(N, q^{l}\right), j\left(q^{l}\right)\right)_{l=1, \ldots, k}$.

Let $z$ be a core allocation in game $(N, v)$ that allows game $v$ to have an extendable core with respect to the list of pairs $\left(\left(N, q^{l}\right), j\left(q^{l}\right)\right)_{l=1, \ldots, k}$. Adding the pair $((N, w), i(w))$ to the list does not change this fact since the allocation $x=z+\left(\frac{w(N)-v(N)}{|N|}, \ldots, \frac{w(N)-v(N)}{|N|}\right)$ is a core allocation in game $w$ and satisfies $x i \geq z_{i}$ for all $i \in N$.

It is well-known that in the class of balanced games, convex games and veto balanced games the nucleolus does not satisfy N-monotonicity. (See Hokari (2000) for the case of convex games and Arin and Feltkamp (2005) for the case of veto balanced games.)

Corollary 10 In the class of balanced games, convex games and veto balanced games the nucleolus is not core monotonic.

The per capita nucleolus is a core concept that satisfies N-monotonicity in the class of all balanced games, which is why some authors consider it a good candidate when seeking to select monotonic core allocations. The following result shows that the per capita nucleolus violates core monotonicity and therefore, even if it does satisfy N-monotonicity, it can hardly be seen as a monotonic core concept (at least in the class of all balanced games and in the subclass of veto balanced games). 
Proposition 11 In the class of balanced games the per capita nucleolus is not core monotonic.

Proof. Let $N=\{1,2,3.4 .5,6\}$ a set of players and consider the following 6 -person veto balanced games:

$$
\begin{aligned}
& v(S)=\left\{\begin{array}{cc}
4 & \text { if } 1 \in S \text { and }|S|=5 \\
10 & \text { if } S=N \\
0 & \text { otherwise }
\end{array}\right. \text { and } \\
& w(S)=\left\{\begin{array}{cc}
8 & \text { if } S=N \backslash\{6\} \\
12 & \text { if } S=N \\
v(S) & \text { otherwise }
\end{array}\right.
\end{aligned}
$$

The per capita nucleolus selects the allocation $(5,1,1,1,1,1)$ in the first game and in the second game selects $\left(\frac{86}{21}, \frac{38}{21}, \frac{38}{21}, \frac{38}{21}, \frac{38}{21}, \frac{14}{21}\right)$. Therefore, the per capita nucleolus is not monotonic since player 1 receives a lower payoff in the second game. The pair $(w, 1)$ is not necessary in a list of pairs in order to make game $v$ a game without an extendable monotonic core with respect to these pairs. If given a list of pairs we have that game $v$ has an extendable monotonic core with respect to that list, adding the pair $(w, 1)$ to the list does not change this fact since the allocation $x=(12,0,0,0,0,0)$ is a core allocation in the game $w$ and satisfies $x_{1} \geq z_{1}$ for all $z \in C(v)$.

Note that the proof is constructed using veto balanced convex games.

Corollary 12 In the class of veto balanced games and in the class of convex games the per capita nucleolus is not core monotonic.

\section{Results and open questions}

The foregoing analysis suggests the following general question:

Problem 13 Are there any core monotonic core concept in the class of balanced games?

It also suggests several other questions concerning classes of balanced games.

In the class of convex games the Shapley value is a core concept and therefore a core monotonic core concept. But, in general, the Shapley value is not a core concept. This motivates the following question: if there are core monotonic core concepts in the class of balanced games do those core concepts coincide with 
the Shapley value in the class of convex games? The answer is given by the following example

Let $N=\{1,2,3.4\}$ a set of players and consider the following 4-person balanced games:

$$
\begin{aligned}
v(S) & =\left\{\begin{array}{cc}
4 & \text { if } S \in\{\{1,2,3\},\{1,2,4\}\{1,3.4\}, N\} \\
0 & \text { otherwise }
\end{array}\right. \text { and } \\
w(S) & =\left\{\begin{array}{cc}
v(S) & \text { if } S \neq N \\
8 & \text { if } S=N
\end{array}\right.
\end{aligned}
$$

The game $w$ is convex and therefore its Shapley value, $\left(3, \frac{5}{3}, \frac{5}{3}, \frac{5}{3}\right)$ is a core allocation. The only core allocation of the game $(N, v)$ is $(4,0,0,0)$. Note that game $w$ is monotonic with respect to game $v$ and any player in $N$. Therefore a monotonic core concept does not select an allocation where player 1 receives a payoff lower than 4 and therefore such a core concept will not coincide with the Shapley value of the game.

In the class of veto balanced games there are core monotonic core concepts (see Arin and Feltkamp (2007)). The solution concepts analyzed in this paper are not defined in the class of balanced games and therefore a similar question can be asked. That is, given a core monotonic concept defined on the class of veto balanced games, say $\phi$, are there any core monotonic core concepts in the class of balanced games coinciding with $\phi$ in the class of veto balanced games?

\section{References}

[1] Arin J and Feltkamp V (2005) Monotonicity properties of the nucleolus on the domain of veto balanced games. TOP 13, 2:331-342

[2] Arin J and V. Feltkamp V (2007) On monotonic core allocations for coalitional games with veto players. Working paper IL2807

[3] Hokari T, (2000), The nucleolus is not aggregate-monotonic on the domain of convex games. Int J of Game Theory 29:133-137

[4] Meggido N (1974) On the monotonicity of the bargaining set, the kernel and the nucleolus of a game. SIAM J of Applied Mathematics 27:355-358

[5] Schmeidler D (1969) The nucleolus of a characteristic function game. SIAM $\mathrm{J}$ of Applied Mathematics 17:1163-1170 
[6] Shapley LS (1953) A Value for n-Person Games in Contributions to the Game Theory, Vol. II Kuhn and Tucker editors

[7] Young HP (1985) Monotonic solutions of cooperative games. Int J of Game Theory 14:65-72 